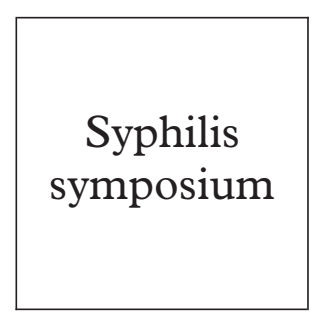

\title{
An outbreak of early syphilis: cases from North Manchester General Hospital
}

\author{
H B Lacey, S P Higgins, D Graham
}

Objectives: To describe the features of an outbreak of early syphilis in North Manchester. Methods: Retrospective KC60 and case note review.

Results: In a 25 month period, 10 cases of primary, 22 of secondary, and nine cases of early latent syphilis were diagnosed. 39 were homosexual or bisexual men, 20 being co-infected with HIV. Most infections were acquired locally and in 19/41 cases oral sex was the only risk factor. High rates of unprotected anal sex were reported among the homosexual men and co-infection with other STIs was common.

Conclusion: This outbreak will facilitate the spread of HIV in the homosexual male population. Unsafe sexual practice is common and the perception that oral sex is "safe" needs revisiting. Delays in accessing GUM clinic appointments must be addressed as part of the control strategy. (Sex Transm Inf 2001;77:311-313)

Keywords: syphilis; HIV; oral sex

\section{Introduction}

This report outlines the demographic and epidemiological details of the patients diagnosed with early syphilis who presented to North Manchester General Hospital. The outbreak of syphilis in Manchester and the surrounding north west started in March 1999 and has been escalating since (by July 2001, over 140 cases in the city of Manchester).

Department of Genitourinary

Manchester General

Hospital, UK

H B Lacey

S P Higgins

D Graham

Correspondence to: Dr H Lacey, Department of GU Medicine, Baillie Street Health Centre, Rochdale OL16 1XS, UK

Accepted for publication 29 August 2001

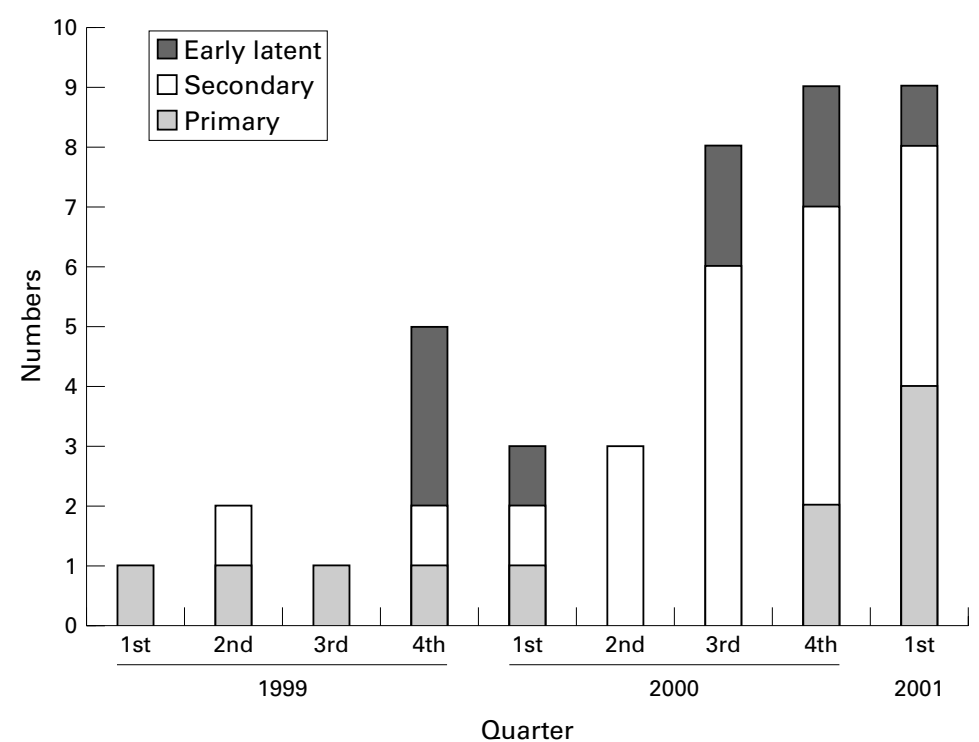

Figure 1 Cases of early syphilis by quarter, North Manchester General Hospital, Fanuary 1999 to end of March 2001. the previous 2 years with no clinical evidence of treponemal infection.

\section{Results}

In the period January 1999 to March 2001, 41 cases of early syphilis were diagnosed (fig 1). Forty cases were male, all were white comprising 36 homosexual, three bisexual, and one heterosexual. Ten patients presented with primary syphilis, two of these had some features of coexistent secondary syphilis (these two referred to as primary cases in the analysis). The most common presenting features were those of secondary syphilis in 22 patients, and nine cases were classed as early latent. The average age was 33 years (range 19-49). Forty patients gave a history of a total of 403 sexual contacts in the preceding 6 months, and one patient gave a personal figure of 480 contacts (total 883 , mean 21.5 per case, range $1-480)$. The patients themselves successfully traced only 27 of these contacts, through social networking. Six had a syphilis diagnosis and five of these are included in this cohort. One case was female, a black Caribbean woman infected through heterosexual sex in Manchester (positive contact seen elsewhere).

The majority of cases were infected in Manchester. Forty of 41 cases had been sexually active (24 of these exclusively) in Manchester within the previous 6 months. One case was probably infected abroad. Eighteen of 41 presented to the walk-in GUM clinic, 10/41 from the HIV treatment clinics in the infectious disease department, 5/41 as a result of partner notification, and eight from GP and other sources.

The following co-infections were diagnosed on routine sexually transmitted infection (STI) screening at presentation. Three patients had rectal gonorrhoea, six chlamydial infection (polymerase chain reaction (PCR) testing, Cobas Amplicor), three non-gonococcal nonchlamydial urethritis, three genital warts, one 


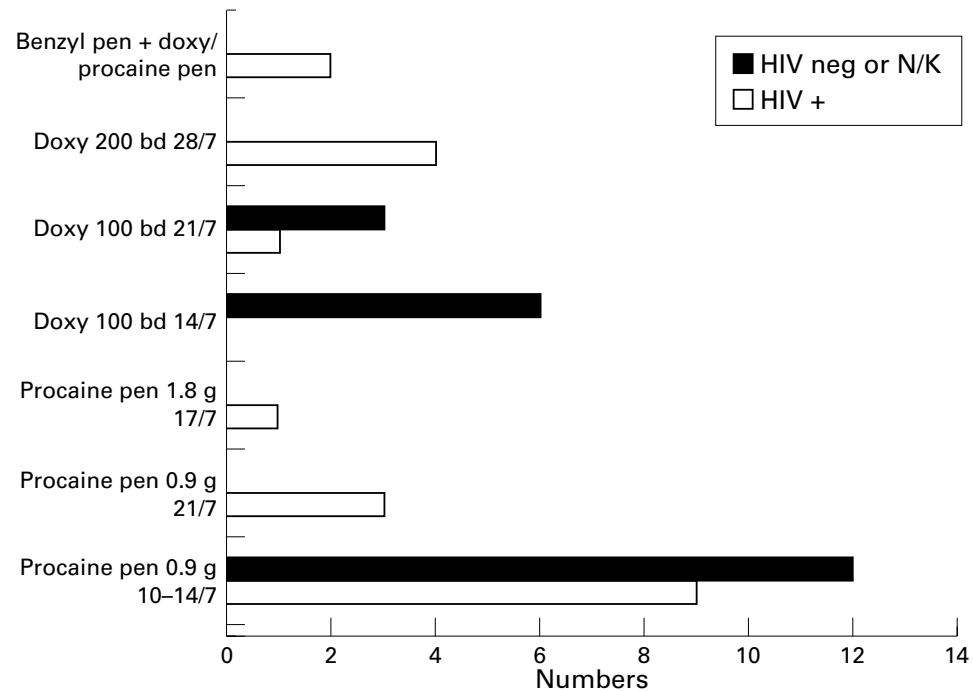

Figure 2 Treatment regimens, 41 cases of early syphilis North Manchester General Hospital.

acute genital herpes simplex (HSV), and one patient had acute hepatitis A virus infection. The patient's HIV status, at the time of syphilis diagnosis, was known in 37/41 cases. Seventeen were HIV negative and 20 were HIV positive, 10 receiving antiretroviral therapy. HIV viral load results at the time of syphilis diagnosis were not available but most patients were not particularly immunosuppressed, the mean CD4 lymphocyte count within 3 months of syphilis diagnosis was 470 (range 96-1000) $\times 10^{6} / 1$.

The risk factor for infection was unprotected oral sex alone in 19/39 homosexual or bisexual men and unprotected oral and anal sex in $19 / 39,10$ of these being HIV positive. One man gave a history of $100 \%$ condom use with oral and anal sex within the time frame of possible infection.

Although none of the patients presented with neurological symptoms or signs, four HIV positive patients had pretreatment lumbar punctures. The EIA was negative in one (no further tests were done on this sample) and positive in three, RPR was negative in all three. TPHA was negative in one, $1 / 1$ in another, and $1 / 80$ in a sample which was a bloody tap (serum TPHA 1/80).

A variety of treatment regimens were used with the minimum course being 10 days of $0.9 \mathrm{~g}$ procaine penicillin daily or doxycycline $200 \mathrm{mg}$ daily for 14 days. The variations reflect the decisions of individual clinicians, with a trend towards longer and higher doses for HIV positive patients, and some patients refused parenteral therapy and opted for oral treatment (fig 2). Benzylpenicillin $1.2 \mathrm{~g}$ intravenously four times daily for 5 days followed by doxycycline or procaine penicillin was used to treat two HIV positive patients under the care of the infectious disease physicians.

Follow up is currently ongoing with 22/41 patients attending for serological monitoring. One HIV negative patient was presumed to be reinfected at 9 months after treatment with 2 weeks of procaine penicillin, following sex with a partner with primary syphilis (RPR had become negative by 6 months, $>1$ in 16 at 9 months). Despite active recall $19 / 41$ patients defaulted follow up, five of these are HIV positive and also defaulted from their HIV appointments. Seven of 19 never returned after treatment, a further nine defaulted after the 3 month visit, and three after the 6 month visit.

\section{Discussion}

Most of these infections were acquired locally in an age group unlikely to have missed HIV prevention messages over the past 15 years. Oral sex is perceived as safer or "safe," which in the case of syphilis acquisition it clearly is not. Although at North Manchester we have not yet documented any coincident HIV seroconversions it is likely that the lesions of primary and secondary syphilis facilitate HIV transmission through oral contact. ${ }^{1}$

Many of the cases of primary syphilis presented with painful multiple genital sores more typical of HSV. The high rate of chlamydial and/or gonococcal co-infection (nine cases in eight patients, seven being rectal infections), suggests a high level of unsafe sexual activity, although five of these patients denied any unprotected anal sex in the previous 6 months. Dark field microscopy was attempted on specimens taken from primary and mucosal lesions of secondary syphilis but the results were negative or inconclusive and probably reflected the lack of experience of medical laboratory scientific officers and clinic medical staff in this technique.

The default rate raises concerns, as $17 \%$ never returned after treatment. This might influence us to favour parenteral regimens as treatment is directly observed. Two of those who never returned received oral treatment and compliance is therefore questionable.

The high rate of syphilis and HIV coinfection will increase concerns about the spread of HIV as STIs, particularly genital ulcerative STIs, are well recognised to facilitate HIV transmission. ${ }^{2}$ Rapid access to treatment is a key element of STI control ${ }^{3}$ yet there have been no improvements in GUM services despite the delays in accessing GUM appointments in the north west being acknowledged to be among the worst in the United Kingdom. ${ }^{4}$

The lack of success in locating and treating contacts results from the casual and often multiple nature of the sexual contacts. Multidisciplinary meetings with public health specialists, GUM consultants, voluntary and non-statutory gay men's agencies were convened in February 2000 and a campaign was launched in March 2000 to raise syphilis awareness in Manchester's "gay" village and among healthcare professionals. Initiatives to promote testing to detect those with early latent disease are needed in appropriate locations including HIV treatment clinics. August bank holiday weekend sees the "gayfest," an event where homosexual men from all over the United Kingdom and further afield converge on Manchester. This event coinciding with an uncontrolled outbreak of syphilis will have implications not only for local 
services but also for those throughout the United Kingdom.

Conflict of interest: none. Source of funding: none.

Source of funding: none.
Contributors: HL contributed to the data collection, carried out Contributors: HL contributed to the data collection, carried out the data analysis, and wrote the first and final draft of the paper; ings and writing the paper; DG contributed to the data collection ings and writing the paper; DG contributed to the data
and edited the method and results section of the paper.
1 Edwards S, Carne C. Oral sex and the transmission of viral STIs. Sex Transm Inf 1998;74:6-10.

2 Fleming DT, Wasserheit JN. From epidemiological synergy to public health policy and practice: the contribution of other sexually transmitted diseases to sexual transmission of HIV infection. Sex Transm Inf 1999;75:3-17.

3 Catchpole M. Sexually transmitted infections: control strategies. BM7 2001;322:1135-6.

4 Foley E, Patel R, Green N, et al. Access to genitourinary medicine clinics in the United Kingdom. Sex Transm Inf 2001;77:12-14 